\title{
Clinical predictors of achieving the minimal clinically important difference after surgery for cervical spondylotic myelopathy: an external validation study from the Canadian Spine Outcomes and Research Network
}

\author{
Nathan Evaniew, MD, PhD, FRCSC, ${ }^{1}$ David W. Cadotte, MD, PhD, FRCSC, ${ }^{1}$ \\ Nicolas Dea, MD, MSc, FRCSC, ${ }^{2}$ Christopher S. Bailey, MD, MSc, FRCSC, ${ }^{3}$ \\ Sean D. Christie, MD, FRCSC, ${ }^{4}$ Charles G. Fisher, MD, MHSc, FRCSC, ${ }^{2}$ \\ Jerome Paquet, MD, FRCSC, ${ }^{5}$ Alex Soroceanu, MD, CM, MPH, FRCSC,1 \\ Kenneth C. Thomas, MD, MHSc, FRCSC, ${ }^{1}$ Y. Raja Rampersaud, MD, FRCSC, ${ }^{6}$ \\ Neil A. Manson, MD, FRCSC, ${ }^{4,7}$ Michael Johnson, MD, FRCSC, ${ }^{8}$ Andrew Nataraj, MD, MSc, FRCSC, ${ }^{9}$ \\ Hamilton Hall, MD, FRCSC, ${ }^{6}$ Greg McIntosh, MSc, ${ }^{10}$ and W. Bradley Jacobs, MD, FRCSC ${ }^{1}$ \\ ${ }^{1}$ University of Calgary Spine Program, University of Calgary, Alberta; ${ }^{2}$ Vancouver Spine Surgery Institute, University of British \\ Columba, Vancouver, British Columbia; ${ }^{3}$ Department of Surgery, Western University, London, Ontario; ${ }^{2}$ Department of Surgery, \\ Dalhousie University, Halifax, Nova Scotia; ${ }^{5}$ Département de chirurgie, Université Laval, Québec; ${ }^{6}$ Department of Surgery, \\ University of Toronto, Ontario; ${ }^{7}$ Canada East Spine Centre, Saint John, New Brunswick; ${ }^{8}$ Department of Surgery, University \\ of Manitoba, Winnipeg, Manitoba; ${ }^{9}$ Department of Surgery, University of Alberta, Edmonton, Alberta; and ${ }^{10}$ Canadian Spine \\ Outcomes and Research Network, Markdale, Ontario, Canada
}

OBJECTIVE Recently identified prognostic variables among patients undergoing surgery for cervical spondylotic myelopathy (CSM) are limited to two large international data sets. To optimally inform shared clinical decision-making, the authors evaluated which preoperative clinical factors are significantly associated with improvement on the modified Japanese Orthopaedic Association (mJOA) scale by at least the minimum clinically important difference (MCID) 12 months after surgery, among patients from the Canadian Spine Outcomes and Research Network (CSORN).

METHODS The authors performed an observational cohort study with data that were prospectively collected from CSM patients at 7 centers between 2015 and 2017. Candidate variables were tested using univariable and multiple binomial logistic regression, and multiple sensitivity analyses were performed to test assumptions about the nature of the statistical models. Validated mJOA MCIDs were implemented that varied according to baseline CSM severity.

RESULTS Among 205 patients with CSM, there were 64 (31\%) classified as mild, 86 (42\%) as moderate, and 55 (27\%) as severe. Overall, $52 \%$ of patients achieved MCID and the mean change in mJOA score at 12 months after surgery was $1.7 \pm 2.6$ points $(p<0.01$ ), but the subgroup of patients with mild CSM did not significantly improve (mean change $0.1 \pm$ 1.9 points, $p=0.8$ ). Univariate analyses failed to identify significant associations between achieving MCID and sex, BMI, living status, education, smoking, disability claims, or number of comorbidities. After adjustment for potential confounders, the odds of achieving MCID were significantly reduced with older age (OR 0.7 per decade, 95\% $\mathrm{Cl} 0.5-0.9$, p < 0.01 ) and higher baseline mJOA score (OR 0.8 per point, $95 \% \mathrm{Cl} 0.7-0.9, \mathrm{p}<0.01$ ). The effects of symptom duration (OR 1.0 per additional month, $95 \% \mathrm{Cl} \mathrm{0.9-1.0,} \mathrm{p}=0.2$ ) and smoking (OR $0.4,95 \% \mathrm{Cl} 0.2-1.0, \mathrm{p}=0.06)$ were not statistically significant.

CONCLUSIONS Surgery is effective at halting the progression of functional decline with CSM, and approximately half of all patients achieve the MCID. Data from the CSORN confirmed that older age is independently associated with poorer outcomes, but novel findings include that patients with milder CSM did not experience meaningful improvement,

ABBREVIATIONS CSM = cervical spondylotic myelopathy; $C S O R N=$ Canadian Spine Outcomes and Research Network; IQR = interquartile range; $M C I D=$ minimum clinically important difference; $\mathrm{mJOA}=$ modified Japanese Orthopaedic Association; NDI = Neck Disability Index

SUBMITTED December 15, 2019. ACCEPTED February 18, 2020

INCLUDE WHEN CITING Published online April 10, 2020; DOI: 10.3171/2020.2.SPINE191495. 
and that symptom duration and smoking were not important. These findings support a nuanced approach to shared decision-making that acknowledges some prognostic uncertainty when weighing the various risks, benefits, and alternatives to surgical treatment.

https://thejns.org/doi/abs/10.3171/2020.2.SPINE191495

KEYWORDS spine; surgery; myelopathy; cervical; outcomes; predictors

$\mathrm{C}$ ERVICAL spondylotic myelopathy (CSM) is the most common etiology of atraumatic spinal cord dysfunction. Patients with CSM must often decide whether or not to undergo decompressive surgery. ${ }^{1,2}$ Current literature supports the idea that surgery for CSM is effective at halting symptom progression, but there is ongoing uncertainly about the extent to which individual patients will experience meaningful improvement. To facilitate shared clinical decision-making, several recent studies have attempted to identify reliable prognostic factors. ${ }^{3-6}$

The current best available evidence comes from Tetreault et al.'s analysis of 743 patients with CSM who were enrolled in two prospective observational studies. This report noted that younger age, shorter duration of symptoms, nonsmoking status, and lack of significant gait impairment were each associated with improvements on the modified Japanese Orthopaedic Association (mJOA) scale by at least the minimum clinically important difference (MCID). The MCID, also referred to as a minimal important difference, is the smallest treatment effect that informed patients are likely to perceive as beneficial (or harmful) enough to justify changes in management. ${ }^{89} \mathrm{Al}-$ though the findings of the Tetreault et al. study are supported by a series of papers sharing the same data set, they have not yet been reproduced in an independent data set. ${ }^{10-12}$

The process of independently verifying novel research findings with new data is referred to as external validation, and it is an imperative part of the scientific method. ${ }^{13}$ External validation studies can evaluate whether promising results are sufficiently dependable to implement in clinical practice and thereby confidently inform the practice of evidence-based medicine. ${ }^{14}$ Whereas the vast majority of research about prognostic factors focuses on model development, external validation studies hold unique potential to bridge critical knowledge gaps. ${ }^{15}$ Therefore, our primary objective in this study was to determine which preoperative clinical factors are significantly associated with improvement on the mJOA scale by at least the MCID among patients in the Canadian Spine Outcomes and Research Network (CSORN) 12 months after surgery for CSM. We specifically aimed to determine whether previously reported predictors had reproducible effects in our unique data set.

\section{Methods}

We performed an observational study that was part of an ongoing multicenter study utilizing the CSORN platform. ${ }^{1,2}$ Patients diagnosed with CSM were enrolled by participating orthopedic and neurosurgical spine surgeons at 7 centers in Canada between 2015 and 2017 (University of Calgary, Université Laval [Québec], Dalhousie University [Halifax], University of Alberta [Edmonton], University of British Columbia [Vancouver], Western University [London], and University of Manitoba [Winnipeg]). Local research coordinators collected data from patients at each site, which were then tracked and audited by a national coordinator. Privacy and security were maintained according to a framework that includes a governance structure, standard operating procedures, training processes, physical and technical security, and privacy impact assessments. We obtained local Clinical Research Ethics Board approval at each participating site prior to enrolling patients, collecting data, and performing this study. All patients provided written informed consent to participate.

\section{Patient Sample}

We included all patients who met eligibility criteria, consented to participate, underwent surgery, and completed 12 months of follow-up. We diagnosed CSM when patients had clinical signs and symptoms of cervical myelopathy with concordant MRI findings of spinal cord compression and/or signal change secondary to spondylosis. We excluded patients with anterior horn disease, multiple sclerosis, tumors, intradural pathology, previous cervical spine surgery, or acute trauma. We did not standardize surgical indications or any of the technical aspects of surgery for this study; all procedures were performed according to the recommendations and preferences of the local surgeons at each site.

\section{Data Sources}

We collected the following baseline characteristics using standardized case report forms: sex, age, BMI, living arrangement (alone vs not alone), education (postsecondary vs less than postsecondary), smoking status, number of comorbidities, presence of disability or worker's compensation claims, and duration of symptoms.

Our primary outcome measure was the mJOA scale, which is an 18-point investigator-administered instrument that measures neurological function and symptoms in patients with CSM. ${ }^{16}$ The mJOA scale has been extensively validated and has been shown to have suitable psychometric properties for evaluating outcomes after surgery for CSM ${ }^{17}$ According to recent analyses by Tetreault et al., the MCID of the MJOA varies with patients' baseline impairment: patients with scores of 15 or greater are considered to have mild myelopathy and their MCID is 1 point, patients with scores of 12-14 are considered to have moderate myelopathy and their MCID is 2 points, and patients with scores of 11 or less are considered to 
have severe myelopathy and their MCID is 3 points. ${ }^{7,16,18}$ MCID is achieved when patients improve by at least the MCID for their specific subgroup of baseline severity. For example, a patient with a baseline mJOA score of 9 must improve by at least 3 points to achieve MCID, while a patient with a baseline mJOA score of 16 must only improve by at least 1 point.

\section{Statistical Analysis}

We report discrete variables as counts or proportions, normally distributed continuous variables as means with standard deviations, and skewed continuous variables as medians with interquartile ranges (IQRs). We used parametric tests for data with normal distributions and nonparametric tests for data without normal distributions. We compared means using two-tailed Student t-tests and proportions with chi-square or Fisher's exact tests.

We evaluated potential predictors of achieving MCID using binomial logistic regression. Candidate variables were initially selected based on clinical relevance and results from the recent literature, then tested with univariate analyses. Variables with $\mathrm{p}$ values $<0.2$ or previously established clinical importance (age, smoking, symptom duration) were included in final multiple logistic regression analyses. We performed multiple sensitivity analyses in which categorical and continuous versions of variables were exchanged where possible, baseline disease severity was considered ordinal (mild, moderate, or severe) rather than scalar, patients with mild CSM were excluded from the analyses, patients with baseline mJOA scores of 18 were excluded, study site was controlled for, and Poisson regression via generalized linear modeling was implemented instead of binomial logistic regression. Poisson regression is not ideal for dichotomous outcomes such as the occurrence of MCID because it assumes a statistical distribution based on counts, ${ }^{19}$ but modified Poisson regression was used previously by other investigators. ${ }^{12} \mathrm{We}$ also performed sensitivity analyses in which alterative multiple logistic regression model-building strategies were tested (full model, backward elimination, and forward selection).

We performed a complete case analysis in which patients with missing data were excluded and imputations were not performed. We tested for multicollinearity using variance inflation factors and we removed or combined independent variables that were highly correlated. We evaluated model fit using the coefficient of determination $\left(\mathrm{R}^{2}\right)$. All tests of significance were two-tailed and $\mathrm{p}$ values $<0.05$ were considered statistically significant. We performed our analyses with IBM SPSS (version 25.0.0.1, IBM Corp.) and Microsoft Excel 2011 (Microsoft Corp.).

\section{Results}

We identified 285 patients who consented, enrolled, underwent surgery for CSM, and achieved at least 12 months of clinical follow-up. We excluded 80 of these because of incomplete baseline or follow-up mJOA data, but their baseline demographics did not differ substantially from our included cohort (Appendix 1). This yielded a final sample of 205 patients for our complete case analysis. We report their baseline demographics in Table 1. The mean
TABLE 1. Baseline demographics of 205 patients with CSM

\begin{tabular}{lc}
\hline \multicolumn{1}{c}{ Variable } & Value \\
\hline Mean age (SD), yrs & $59.5(11.8)$ \\
\hline Males (\%) & $124(60)$ \\
\hline Mean BMI (SD), kg/m² & $28.5(6.4)$ \\
\hline Lives alone (\%) & $32(16)$ \\
\hline Post-secondary education or greater (\%) & $92(45)$ \\
\hline Current smoker (\%) & $29(14)$ \\
\hline Current disability claims (\%) & $29(14)$ \\
\hline Mean no. of comorbidities (SD) & $2.9(1.9)$ \\
\hline Mean duration of symptoms (\%), mos & $15.1(9.3)$ \\
\hline$<6$ wks & $5(2)$ \\
\hline 6 wks to 3 mos & $10(5)$ \\
\hline $3-6$ mos & $24(12)$ \\
\hline $6-12$ mos & $38(19)$ \\
\hline $1-2$ yrs & $25(12)$ \\
\hline$>2$ yrs & $103(50)$ \\
\hline Mean baseline mJOA score (SD) & $13.0(2.7)$ \\
\hline Mild (15-18), $\mathrm{n}(\%)$ & $64(31)$ \\
\hline Moderate (12-14), $\mathrm{n}(\%)$ & $86(42)$ \\
\hline Severe (0-11), $\mathrm{n}(\%)$ & $55(27)$ \\
\hline
\end{tabular}

patient age was $59.5 \pm 11.8$ years, $60 \%$ were male, and $14 \%$ were current smokers. Among smokers, the mean number of cigarettes consumed daily was $13.6 \pm 10.0$. Duration of symptoms ranged from less than 6 weeks to greater than 2 years, with a mean of $15.1 \pm 9.3$ months. Half of the cohort (50\%) had a preoperative symptom duration of more than 2 years. Among patients with complete surgical data (n $=155,76 \%$ ), approaches included anterior alone in 44\%, posterior alone in 53\%, and combined anterior-posterior in $3 \%$.

As shown in Fig. 1, our cohort's mean mJOA score at baseline (prior to surgery) was $13.0 \pm 2.7$. There were 64 patients $(31 \%)$ classified as mild, $86(42 \%)$ as moderate, and $55(27 \%)$ as severe. At 12 months after surgery, we observed a statistically significant improvement in mJOA score across our cohort (mean mJOA score $14.7 \pm 2.4$, p < 0.01 ). This finding was consistent for subgroups of patients with moderate (mean change $1.7 \pm 2.2, \mathrm{p}<0.01$ ) and severe (mean change $3.5 \pm 2.6, \mathrm{p}<0.01) \mathrm{CSM}$; however, the subgroup of patients with mild CSM did not significantly improve (mean change $0.1 \pm 1.9, \mathrm{p}=0.8$; Fig. 2). One hundred seven patients (52\%) experienced improvements that were equal to or greater than the MCID for their subgroup of baseline severity. The proportion of patients with mild CSM that achieved MCID (41\%) was significantly less than those with either moderate $(51 \%)$ or severe $(67 \%)$ CSM (chi-square $=8.48$, degrees of freedom $=2, p=0.01$; Fig. 3). The proportions of patients that experienced the MCID according to decade of age are shown in Fig. 4.

Univariate binomial logistic regression analyses failed to identify significant unadjusted associations between MCID and sex, BMI, living status, education, smoking, disability claims, or number of comorbidities (Table 2). 


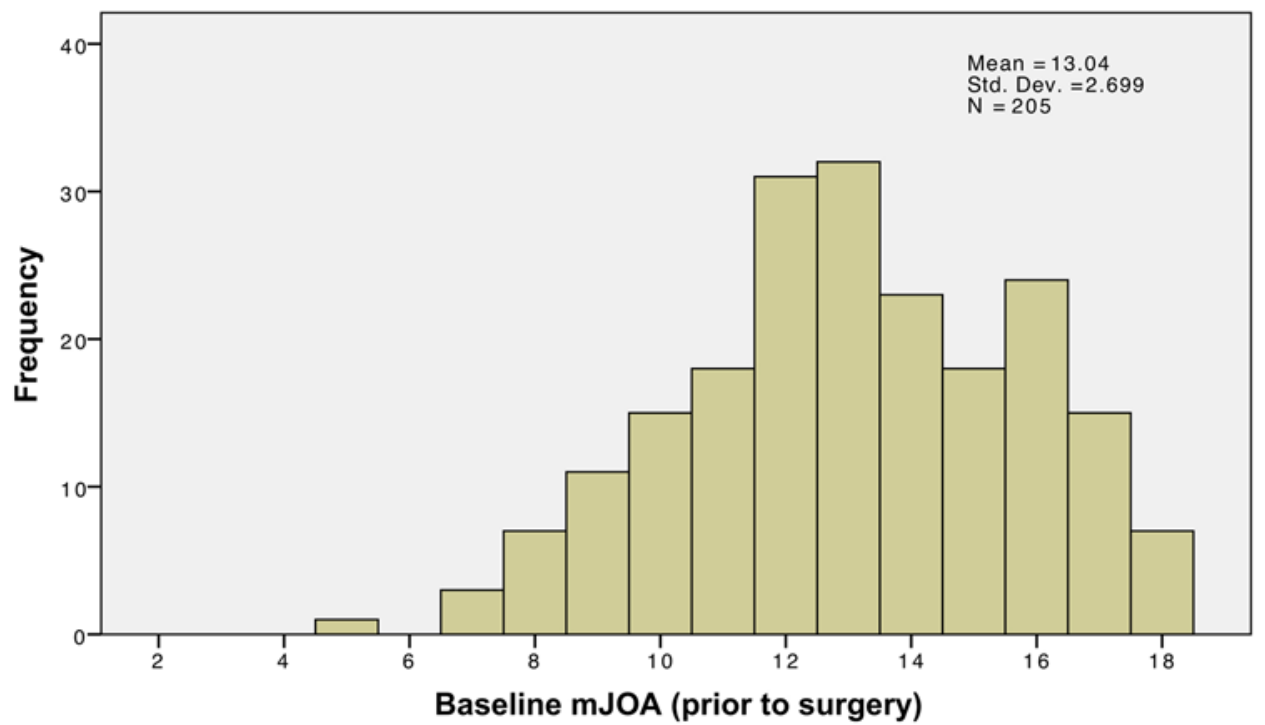

FIG. 1. Distribution of baseline mJOA scores prior to surgery among 205 patients with mild (mJOA score > 15), moderate (score $12-14)$, or severe (score $0-11$ ) CSM. Figure is available in color online only.

However, age (OR 0.84 per additional decade, 95\% CI $0.67-1.05, \mathrm{p}=0.13$ ) and duration of symptoms (OR 0.97 per additional month, 95\% CI 0.94-1.00, $\mathrm{p}=0.03$ ) each met our threshold for inclusion in the adjusted analyses. Furthermore, the unadjusted odds of achieving MCID according to baseline mJOA score (OR 0.81 per additional point, 95\% CI $0.73-0.91, \mathrm{p}<0.01)$ or worse category of baseline severity (OR 1.72, 95\% CI 1.19-2.50, p < 0.01) were both statistically significant.

Our final multiple binomial logistic regression model is shown in Table 3. After adjustment, the odds of achieving MCID was statistically significantly reduced with older

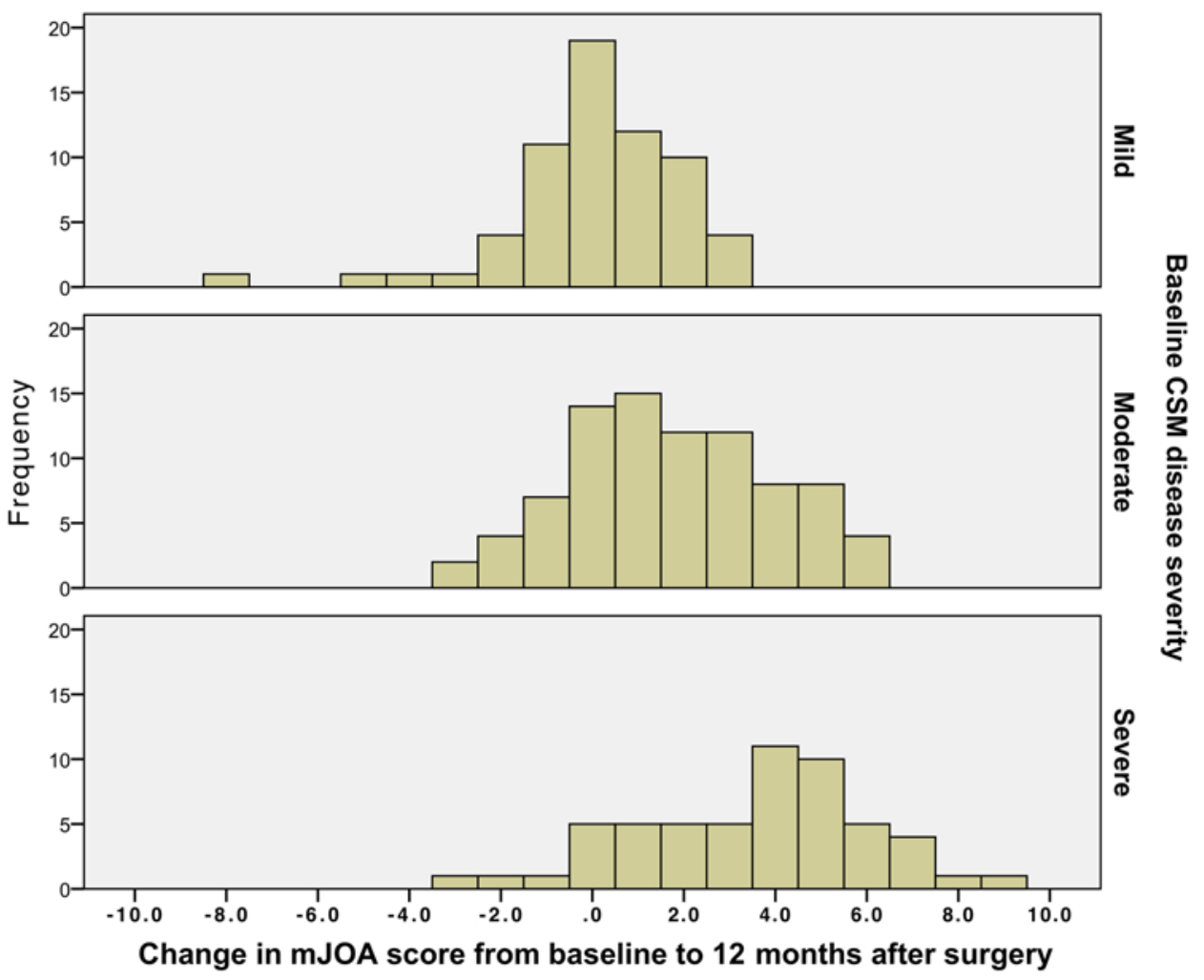

FIG. 2. Changes in mJOA scores at 12 months after surgery among patients with mild $(n=64)$, moderate $(n=86)$, and severe $(n=55)$ CSM. Positive change scores indicate improvement in myelopathy symptoms. Figure is available in color online only. 


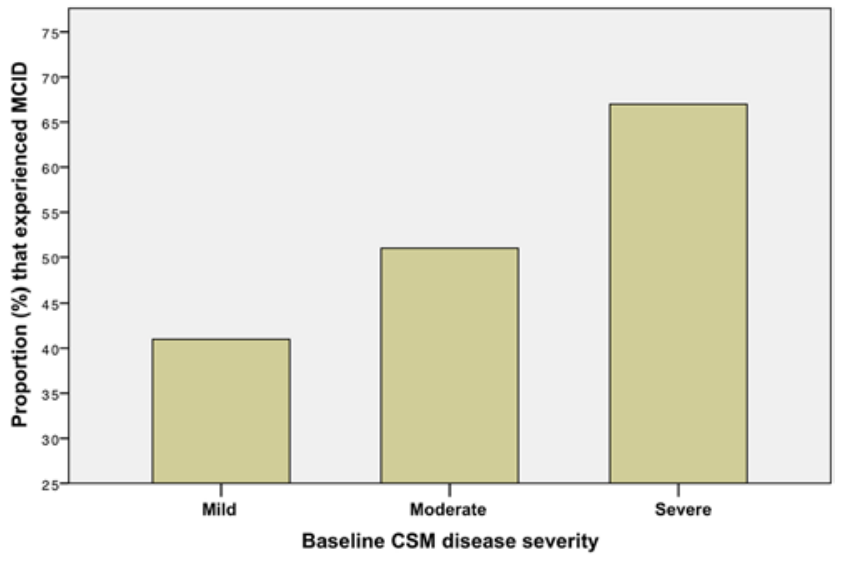

FIG. 3. Proportions of patients with mild $(n=64)$, moderate $(n=86)$, and severe $(n=55)$ CSM that experienced the MCID at 12 months after surgery. The proportion of patients with mild CSM that experienced MCID (41\%) was significantly less than those with either moderate $(51 \%)$ or severe $(67 \%$ ) CSM (chi-square $=8.48$, degrees of freedom $=2, p=$ $0.01)$. Figure is available in color online only.

age (OR 0.67 per decade, 95\% CI $0.53-0.89, \mathrm{p}<0.01$ ) and higher baseline mJOA score (OR 0.76 per point, $95 \% \mathrm{CI}$ $0.70-0.86, \mathrm{p}<0.01)$. The effects of symptom duration (OR 0.98 per additional month, 95\% CI $0.95-1.01, \mathrm{p}=0.24$ ) and current smoking status (OR 0.43 among smokers, 95\% CI $0.18-1.04, \mathrm{p}=0.06)$ were not statistically significant. Overall model fit was modest $\left(\mathrm{R}^{2}=0.17\right)$. These findings were consistent across sensitivity analyses in which categorical and continuous versions of variables were substituted where possible, baseline disease severity was considered ordinal rather than scalar, patients with mild CSM were excluded, patients with baseline mJOA scores of 18 $(n=7)$ were excluded (Appendix 2), study site was controlled for, Poisson regression was implemented instead of binomial logistic regression, and alternative strategies of model building were implemented.

\section{Discussion}

We performed an observational cohort study to determine which preoperative clinical factors were significantly associated with improvement on the mJOA scale by at least the MCID at 12 months among CSORN patients who underwent surgical management for CSM. We found that older age was a negative prognostic factor, which confirmed prior literature, but we failed to confirm significant independent associations for symptom duration and smoking status. We also found that patients with mild CSM were significantly less likely to experience an MCID compared to those with moderate or severe CSM, which manifested as higher baseline mJOA score being significantly and independently associated with decreased odds of achieving the MCID.

\section{Strengths and Limitations}

The major strength of this study is our use of the CSORN data set to test the external validity of previously reported clinical predictors of MCID. In comparison to

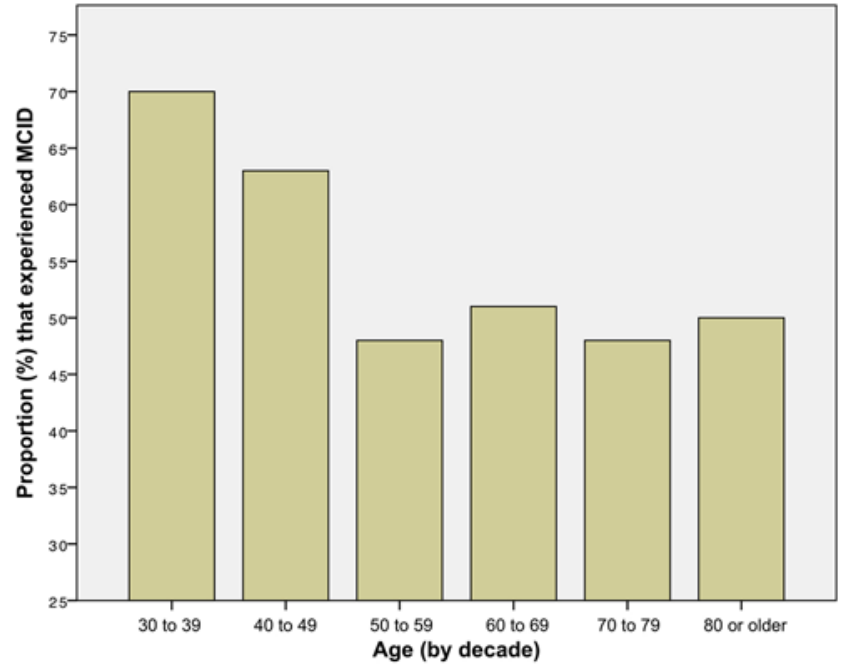

FIG. 4. Proportions of patients with CSM that experienced the MCID at 12 months after surgery according to decade of age. Figure is available in color online only.

the cohort of patients reported by Tetreault et al. in their analysis of the AOSpine North America and International data sets, ${ }^{7}$ our patients in the CSORN consisted of an independent prospective sample with relatively similar distributions of age, sex, smoking status, symptom duration, and baseline mJOA scores. Our study is also strengthened by our use of conventional statistical techniques and implementation of multiple sensitivity analyses.

Nonetheless, we note several important limitations. First, our data forms were not designed to purposefully replicate those of prior studies, which meant that we could not test all of the candidate variables reported in the prior literature. Specifically, we did not have identical measures (or reasonable surrogates) of the specific comorbidities, pathological diagnoses, and clinical signs and symptoms that Tetreault et al. reported on. ${ }^{7}$ Therefore, we were unable to evaluate the calibration or discrimination of their model in our data set. These are useful metrics in external validation studies of prognostic models because they quantify performance and show relations between observed and expected outcomes. ${ }^{20} \mathrm{We}$ instead performed a de novo analysis of the candidate variables that were available in our data set, a strategy that favored pragmatism and feasibility.

Second, our final eligible cohort of 205 is considerably smaller than the cohort of 743 patients in the study of Tetreault et al., ${ }^{7}$ and we had to exclude 80 patients because of incomplete baseline or follow-up mJOA scale data. However, we did identify that the baseline demographics of our excluded patients did not differ substantially from our included patients, which suggests that specific selection bias related to missingness is unlikely. Nonetheless, similarity of baseline characteristics between missing and included cases does not completely address the concern that postoperative outcomes might be different. It is therefore plausible that bias toward misleading results could still exist and that confidence in our results might be limited. It would have been preferable if our entire study sample had 
TABLE 2. Univariate binomial logistic regression showing unadjusted associations between candidate variables and achievement of the MCID among patients with mild, moderate, or severe CSM

\begin{tabular}{lccc}
\hline \multicolumn{1}{c}{ Variable } & OR & $95 \% \mathrm{Cl}$ & $\begin{array}{c}\mathrm{p} \\
\text { Value }\end{array}$ \\
\hline Age (per additional decade) & 0.84 & $0.67-1.05$ & 0.13 \\
\hline Sex (male) & 0.98 & $0.56-1.72$ & 0.95 \\
\hline BMI & 1.02 & $0.97-1.07$ & 0.36 \\
\hline Living status (lives alone) & 1.21 & $0.57-2.60$ & 0.62 \\
\hline Education (post-secondary or greater) & 0.92 & $0.53-1.61$ & 0.77 \\
\hline Smoking status (current smoker) & 0.60 & $0.27-1.33$ & 0.21 \\
\hline Current disability claims & 0.71 & $0.32-1.56$ & 0.39 \\
\hline No. of comorbidities & 0.98 & $0.84-1.14$ & 0.78 \\
\hline Symptom duration (per additional month) & 0.97 & $0.94-1.00$ & 0.03 \\
\hline Baseline mJOA score (per additional point) & 0.81 & $0.73-0.91$ & $<0.01$ \\
\hline
\end{tabular}

achieved complete follow-up, and this observation will guide the design and conduct of future CSORN studies to maximize data capture.

Likewise, conventional sample size formulae suggest post hoc that our final model had sufficient power to detect at least medium effect sizes among four predictor variables. ${ }^{21,22}$ There are a variety of modern statistical techniques available to impute missing data, but we ultimately chose to forego those approaches because all involve assumptions about the nature of missingness that are critically and fundamentally untestable. ${ }^{23,24}$ Indeed, there are no truly credible substitutes for data that does not exist and others have supported that complete case analyses should be emphasized whenever possible. ${ }^{25}$ For comparison, Tetreault et al. reported that only $74 \%$ of their sample attended their final follow-up visits and that they imputed follow-up data for 137 participants. They did not report on any methods to mitigate the potential errors and/or biases that were introduced?

Third, our study evaluated candidate variables inherent to patients' clinical presentations alone, which means it does not inform about the potential prognostic ability of various radiological findings or the importance of specific surgical techniques. For example, we did not investigate the role of altered MRI signal intensity, ${ }^{6}$ sagittal cervical malalignment, ${ }^{26}$ or the effect of anterior versus posterior approaches. ${ }^{4}$ We also did not investigate associations with outcomes other than the mJOA score, such as the Neck Disability Index (NDI), Short Form-12, EuroQol-5D, or visual analog scales for neck and arm pain.

Although the mJOA scale is currently the most accepted outcome measure in CSM research, it is investigator-reported rather than patient-reported, and functional improvements on the mJOA may have limited correlation with patient-reported health status. ${ }^{27,28}$ The mJOA is also susceptible to ceiling effects, which may limit its ability to discriminate subtle but important changes in hand function or gait stability among patients with mild disease. This could have reasonably contributed to our observation of less achievement of an MCID among patients with mild
TABLE 3. Multiple binomial logistic regression

\begin{tabular}{lccc}
\hline \multicolumn{1}{c}{ Variable } & OR & $95 \% \mathrm{Cl}$ & $\begin{array}{c}\mathrm{p} \\
\text { Value }\end{array}$ \\
\hline Age (per additional decade) & 0.67 & $0.53-0.89$ & $<0.01$ \\
\hline Smoking status (current smoker) & 0.43 & $0.18-1.04$ & 0.06 \\
\hline Symptom duration (per additional month) & 0.98 & $0.95-1.01$ & 0.24 \\
\hline Baseline mJOA score (per additional point) & 0.76 & $0.70-0.86$ & $<0.01$ \\
\hline
\end{tabular}

Adjusted associations between candidate variables and achievement of the MCID among patients with mild, moderate, or severe CSM. Model fit: $R^{2}=0.17$.

CSM. We included patients with baseline mJOA scores of 18 because they presented with signs and/or symptoms of cervical myelopathy and met our inclusion criteria. However, their baseline scores meant that they could not have achieved any improvement on the mJOA scale. We performed a sensitivity analysis in which these patients $(n=7$, $1.5 \%$ of our cohort) were excluded, and the results did not differ in magnitude or significance from our primary final multivariate model.

\section{Relation to Prior Literature}

Our study adds to a growing body of literature examining function and disability in patients with cervical myelopathy, much of which comes from analyses of the AOSpine International and North America data sets. The severity-based MCID was derived from this data using a combination of distribution-, anchor-, and expertise-based techniques..$^{18}$ Of note, the anchor used was a previously established MCID for the NDI, rather than a concurrent prospective Global Rating of Change. This is an important limitation because the NDI is an instrument for rating the extent to which neck pain affects patients' function and activities rather than the neurological deficits inherent to CSM. ${ }^{29}$ Indeed, Kopjar et al. identified limited correlation between mJOA and NDI scores. ${ }^{17}$ Kato et al. evaluated the MCID of the unmodified JOA score in a retrospective analysis of 101 patients treated with laminoplasty, but reported insufficient power to perform severity-based stratification. ${ }^{30}$

Our finding of greater improvement among patients with worse preoperative disease severity is novel in the CSM literature. Although the common statistical and clinical phenomenon of regression toward a mean could contribute to this observation, it has not been previously reported. In the AOSpine International and North America dataset analyses, worse preoperative disease severity had no association with achievement of MCID (unadjusted relative risk $0.99,95 \%$ CI $0.97-1.01, \mathrm{p}=0.04)^{7}$ but an inverse association with the probability of achieving an mJOA score of 16 or greater (relative risk 1.11, 95\% CI 1.07-1.15, $\mathrm{p}<0.01) .^{12}$ This latter finding is unsurprising because it is intuitive that patients with worse preoperative disease severity would be less likely to reach an unadjusted outcome threshold. Elsewhere, Asher et al. analyzed registry data from 1963 patients who underwent elective surgery for CSM and found that preoperative mJOA score was not associated with 12-month overall satisfaction. ${ }^{31}$ Reports 
on outcomes of patients with cervical deformity and myelopathy from the International Spine Study Group did not adjust for baseline mJOA scores or implement severitybased MCIDs. ${ }^{26,32,33}$

Current clinical practice guidelines recommend surgery for patients with moderate or severe CSM, but acknowledge uncertainty in the management of patients with mild CSM. The 2017 guideline of AOSpine North America and the Cervical Spine Research Society states that patients with mild CSM may be offered either surgical intervention or a supervised trial of structured rehabilitation through a process of shared decision-making. ${ }^{34}$ The strength of this recommendation was graded as "weak" based on "very low to low" quality evidence. However, the guidelines panel "strongly recommended" that patients who experience neurological deterioration during their course of nonoperative treatment should be counseled to undergo surgery, based on evidence that longer duration of symptoms and worse myelopathy severity may be negative prognostic factors. Although it is intuitive to offer surgery when counseling patients with neurological deterioration, our results do not support the importance of these particular prognostic factors. In a recent analysis of the AOSpine International and North America data sets, Badhiwala et al. reported a mean mJOA score improvement of 0.9 points (95\% CI 0.4-1.3 points) among the subgroup of 193 patients with mild CSM at 24 months after surgery; they did not report the proportion that achieved MCID. ${ }^{35} \mathrm{We}$ did not evaluate patients with mild CSM treated nonoperatively in this study, but we support that this group of patients warrants further study.

\section{Implications}

From a clinical point of view, our results suggest that clinicians should proceed cautiously when counseling individual patients about their likelihood of experiencing meaningful neurological recovery after surgery for CSM. Although we confirmed that older age appears to be independently associated with poorer outcomes, we failed to confirm negative associations with worse preoperative disease severity, longer preoperative symptom duration, and smoking status. These findings support a nuanced approach to shared decision-making that acknowledges some prognostic uncertainty when weighing the various risks, benefits, and alternatives to surgical treatment. Our study introduces the novel possibilities that patients with milder CSM may be less likely to experience meaningful improvement in mJOA scores and that symptom duration might not be important.

From a methodological point of view, our study highlights the importance of external validation while supporting that further research is necessary to clarify the reliability of prognostic factors among patients with CSM. Recent reports have described a "reproducibility crisis" in modern academic medicine, in which more than $70 \%$ of researchers have failed to reproduce others' results. ${ }^{36}$ In response, recent advances in research methodology have increased awareness of safeguards against spurious and misleading conclusions. ${ }^{37-39}$ Ultimately, users of evidence must consider the totality of the literature when making treatment decisions, and their confidence should be limited when re- search findings conflict or cannot be reproduced..$^{40,41}$ There are many plausible explanations for varying conclusions across apparently similar studies, but it seems least plausible that there would exist fundamental differences in the underlying biology or pathophysiology of CSM among patients from different cohorts.

\section{Conclusions}

Surgery is effective at halting the progression of functional decline with CSM, and approximately half of all patients achieve the MCID. Data from the CSORN confirmed that older age is independently associated with poorer outcomes, but novel findings include that patients with milder CSM did not experience meaningful improvement, and that symptom duration and smoking were not important. These findings support a nuanced approach to shared decision-making that acknowledges some prognostic uncertainty when weighing the various risks, benefits, and alternatives to surgical treatment.

\section{Acknowledgments}

We thank all of the patients who participated in the study and the support and research coordinator staff and investigators from the CSORN contributing sites.

\section{References}

1. Bond M, McIntosh G, Fisher C, et al. Treatment of mild cervical myelopathy: factors associated with decision for surgical intervention. Spine (Phila Pa 1976). 2019;44(22):16061612.

2. Sharifi B, McIntosh G, Fisher C, et al. Consultation and surgical wait times in cervical spondylotic myelopathy. Can J Neurol Sci. 2019;46(4):430-435.

3. Aggarwal RA, Srivastava SK, Bhosale SK, Nemade PS. Prediction of surgical outcome in compressive cervical myelopathy: a novel clinicoradiological prognostic score. $\mathrm{J} \mathrm{Cra-}$ niovertebr Junction Spine. 2016;7(2):82-86.

4. Kato S, Nouri A, Wu D, et al. Comparison of anterior and posterior surgery for degenerative cervical myelopathy: an MRI-based propensity-score-matched analysis using data from the prospective multicenter AOSpine CSM North America and international studies. J Bone Joint Surg Am. 2017;99(12):1013-1021.

5. Nouri A, Tetreault L, Côté P, et al. Does magnetic resonance imaging improve the predictive performance of a validated clinical prediction rule developed to evaluate surgical outcome in patients with degenerative cervical myelopathy? Spine (Phila Pa 1976). 2015;40(14):1092-1100.

6. Tetreault LA, Dettori JR, Wilson JR, et al. Systematic review of magnetic resonance imaging characteristics that affect treatment decision making and predict clinical outcome in patients with cervical spondylotic myelopathy. Spine (Phila Pa 1976). 2013;38(22)(suppl 1):S89-S110.

7. Tetreault L, Wilson JR, Kotter MRN, et al. Predicting the minimum clinically important difference in patients undergoing surgery for the treatment of degenerative cervical myelopathy. Neurosurg Focus. 2016;40(6):E14.

8. Jaeschke R, Singer J, Guyatt GH. Measurement of health status. Ascertaining the minimal clinically important difference. Control Clin Trials. 1989;10(4):407-415.

9. Schünemann HJ, Guyatt GH. Commentary-goodbye M(C) ID! Hello MID, where do you come from? Health Serv Res. 2005;40(2):593-597.

10. Tetreault LA, Côté P, Kopjar B, et al. A clinical prediction 
model to assess surgical outcome in patients with cervical spondylotic myelopathy: internal and external validations using the prospective multicenter AOSpine North American and international datasets of 743 patients. Spine J. 2015;15(3):388-397.

11. Tetreault LA, Kopjar B, Vaccaro A, et al. A clinical prediction model to determine outcomes in patients with cervical spondylotic myelopathy undergoing surgical treatment: data from the prospective, multi-center AOSpine North America study. J Bone Joint Surg Am. 2013;95(18):1659-1666.

12. Tetreault L, Kopjar B, Côté P, et al. A clinical prediction rule for functional outcomes in patients undergoing surgery for degenerative cervical myelopathy: analysis of an international prospective multicenter data set of 757 subjects. J Bone Joint Surg Am. 2015;97(24):2038-2046.

13. Altman DG, Vergouwe Y, Royston P, Moons KGM. Prognosis and prognostic research: validating a prognostic model. $B M J$. 2009;338:b605.

14. Riley RD, Ensor J, Snell KIE, et al. External validation of clinical prediction models using big datasets from e-health records or IPD meta-analysis: opportunities and challenges. BMJ. 2016;353:i3140.

15. Bouwmeester W, Zuithoff NPA, Mallett S, et al. Reporting and methods in clinical prediction research: a systematic review. PLoS Med. 2012;9(5):1-12.

16. Tetreault L, Kopjar B, Nouri A, et al. The modified Japanese Orthopaedic Association scale: establishing criteria for mild, moderate and severe impairment in patients with degenerative cervical myelopathy. Eur Spine J. 2017;26(1):78-84.

17. Kopjar B, Tetreault L, Kalsi-Ryan S, Fehlings M. Psychometric properties of the modified Japanese Orthopaedic Association scale in patients with cervical spondylotic myelopathy. Spine (Phila Pa 1976). 2015;40(1):E23-E28.

18. Tetreault L, Nouri A, Kopjar B, et al. The minimum clinically important difference of the modified Japanese Orthopaedic Association scale in patients with degenerative cervical myelopathy. Spine (Phila Pa 1976). 2015;40(21):1653-1659.

19. Weaver CG, Ravani P, Oliver MJ, et al. Analyzing hospitalization data: potential limitations of Poisson regression. Nephrol Dial Transplant. 2015;30(8):1244-1249.

20. Steyerberg EW, Vickers AJ, Cook NR, et al. Assessing the performance of prediction models: a framework for traditional and novel measures. Epidemiology. 2010;21(1):128-138.

21. Demidenko E. Sample size and optimal design for logistic regression with binary interaction. Stat Med. 2008;27(1):36-46.

22. Demidenko E. Sample size determination for logistic regression revisited. Stat Med. 2007;26(18):3385-3397.

23. Donders ART, van der Heijden GJMG, Stijnen T, Moons KGM. Review: a gentle introduction to imputation of missing values. J Clin Epidemiol. 2006;59(10):1087-1091.

24. Royston P, Moons KGM, Altman DG, Vergouwe Y. Prognosis and prognostic research: developing a prognostic model. BMJ. 2009;338:b604.

25. Akl EA, Briel M, You JJ, et al. Potential impact on estimated treatment effects of information lost to follow-up in randomised controlled trials (LOST-IT): systematic review. BMJ. 2012;344:e2809.

26. Passias PG, Horn SR, Bortz CA, et al. The relationship between improvements in myelopathy and sagittal realignment in cervical deformity surgery outcomes. Spine (Phila Pa 1976). 2018;43(16):1117-1124.

27. Furlan JC, Craven BC. Psychometric analysis and critical appraisal of the original, revised, and modified versions of the Japanese Orthopaedic Association score in the assessment of patients with cervical spondylotic myelopathy. Neurosurg Focus. 2016;40(6):E6.

28. Tetreault LA, Zhu MP, Howard RM, et al. The discrepancy between functional outcome and self-reported health status after surgery for degenerative cervical myelopathy. Spine J. 2019;19(11):1809-1815.
29. Carreon LY, Glassman SD, Campbell MJ, Anderson PA. Neck Disability Index, short form-36 physical component summary, and pain scales for neck and arm pain: the minimum clinically important difference and substantial clinical benefit after cervical spine fusion. Spine J. 2010;10(6):469474.

30. Kato S, Oshima Y, Matsubayashi Y, et al. Minimum clinically important difference and patient acceptable symptom state of Japanese Orthopaedic Association score in degenerative cervical myelopathy patients. Spine (Phila Pa 1976). 2019;44(10):691-697.

31. Asher AL, Devin CJ, Weisenthal BM, et al. Effect of modified Japanese Orthopedic Association severity classifications on satisfaction with outcomes 12 months after elective surgery for cervical spine myelopathy. Spine (Phila Pa 1976). 2019;44(11):801-808.

32. Passias PG, Horn SR, Oh C, et al. Evaluating cervical deformity corrective surgery outcomes at 1-year using current patient-derived and functional measures: are they adequate? $J$ Spine Surg. 2018;4(2):295-303.

33. Poorman GW, Passias PG, Horn SR, et al. Despite worse baseline status depressed patients achieved outcomes similar to those in nondepressed patients after surgery for cervical deformity. Neurosurg Focus. 2017;43(6):E10.

34. Fehlings MG, Tetreault LA, Riew KD, et al. A clinical practice guideline for the management of patients with degenerative cervical myelopathy: recommendations for patients with mild, moderate, and severe disease and nonmyelopathic patients with evidence of cord compression. Global Spine J. 2017;7(3)(suppl):70S-83S.

35. Badhiwala JH, Witiw CD, Nassiri F, et al. Efficacy and safety of surgery for mild degenerative cervical myelopathy: results of the AOSpine North America and international prospective multicenter studies. Neurosurgery. 2019;84(4):890-897.

36. Baker M. 1,500 scientists lift the lid on reproducibility. $\mathrm{Na}$ ture. 2016;533(7604):452-454.

37. Djulbegovic B, Guyatt GH. Progress in evidence-based medicine: a quarter century on. Lancet. 2017;390(10092):415-423.

38. Evaniew N, Carrasco-Labra A, Devereaux PJ, et al. How to use a randomized clinical trial addressing a surgical procedure: users' guide to the medical literature. JAMA Surg. 2016;151(7):657-662.

39. Atkins D, Best D, Briss PA, et al. Grading quality of evidence and strength of recommendations. BMJ. 2004;328(7454): 1490.

40. Balshem H, Helfand M, Schünemann HJ, et al. GRADE guidelines: 3. Rating the quality of evidence. J Clin Epidemiol. 2011;64(4):401-406.

41. Evaniew N, van der Watt L, Bhandari M, et al. Strategies to improve the credibility of meta-analyses in spine surgery: a systematic survey. Spine J. 2015;15(9):2066-2076.

\section{Disclosures}

Dr. Dea reports direct stock ownership in Medtronic, being a consultant for Stryker, and serving on the speakers bureau for Medtronic and Baxter. Dr. Christie reports being a consultant for Metronic Canada. Dr. Fisher reports being a consultant to Medtronic and NuVasive, receiving royalties from Medtronic, receiving clinical or research support for the study from AOSpine, and receiving fellowship support paid to institution from

Medtronic and AOSpine. Dr. Rampersaud reports receiving royalties from and being a consultant to Medtronic. Dr. Manson reports being a consultant to Medtronic Canada and receiving support of non-study-related clinical or research effort from Medtronic Canada. Dr. Johnson reports receiving financial support from Stryker to help fund his research department, which collects data for the CSS Registry. Dr. Jacobs reports being a consultant to Medtronic, Stryker, and DePuy-Synthes. 


\section{Author Contributions}

Conception and design: Evaniew, Cadotte, Dea, Hall, Jacobs. Acquisition of data: Evaniew, Cadotte, Dea, Bailey, Christie, Fisher, Paquet, Soroceanu, Thomas, Rampersaud, Manson, Johnson, Nataraj, McIntosh, Jacobs. Analysis and interpretation of data: Evaniew, Cadotte, Dea, Jacobs. Drafting the article: Evaniew. Critically revising the article: all authors. Reviewed submitted version of manuscript: all authors. Approved the final version of the manuscript on behalf of all authors: Evaniew. Statistical analysis: Evaniew, Soroceanu. Administrative/technical/material support: Evaniew, Cadotte, McIntosh, Jacobs. Study supervision: Evaniew, Dea, McIntosh, Jacobs.

\section{Supplemental Information}

Online-Only Content

Supplemental material is available with the online version of the article.

Appendices 1 and 2. https://thejns.org/doi/suppl/10.3171/2020. 2.SPINE191495.

\section{Correspondence}

Nathan Evaniew: University of Calgary, Calgary, AB, Canada. nevaniew@gmail.com. 